\title{
INCOMPRESSIBILITY OF PRODUCTS OF WEIL TRANSFERS OF GENERALIZED SEVERI-BRAUER VARIETIES
}

\author{
NIKITA A. KARPENKO
}

\begin{abstract}
We generalize the result of [11] on incompressibility of Galois Weil transfer of generalized Severi-Brauer varieties, to direct products of varieties of such type; as shown in [11], this is needed to compute essential dimension of representations of finite groups. We also provide a generalization to non-Galois (separable) Weil transfer.
\end{abstract}

\section{Contents}

1. Introduction 1

2. Non-Galois extensions 2

3. Incompressibility of products 4

4. Particular products 8

4a. Products of Severi-Brauer varieties 8

4b. Weil transfer of products of Severi-Brauer varieties 10

4c. Weil transfer of products of generalized Severi-Brauer varieties 10

5. Quadratic extensions 11

$\begin{array}{ll}\text { References } & 12\end{array}$

\section{INTRODUCTION}

Let $F$ be a field and let $L / F$ be a finite Galois field extension. A central division $L$ algebra $D$ is balanced, if for every $g \in \operatorname{Gal}(L / F)$, the conjugate $L$-algebra $g(D)$, obtained from $D$ by the base change $g: L \rightarrow L$, is Brauer-equivalent to a tensor power of $D$.

For a balanced $D$ as above, we refer as $\mathcal{R}$ SB-variety to the Weil transfer $\mathcal{R}_{L / F}$ of a generalized Severi-Brauer variety $\mathrm{SB}(j, D)$ of $D(j=1, \ldots$, deg $D)$. Let $p$ be a prime integer. An $\mathcal{R S B}$-variety is a $p \mathcal{R S B}$-variety, if the integers $[L: F]$, deg $D$, and $j$ are $p$-powers.

The following problem has been raised in [11] for needs of computing essential $p$ dimension of a representation of a finite group: compute canonical $p$-dimension of an arbitrary finite direct product of $\mathcal{R} \mathrm{SB}$-varieties. This problem has been solved in [11] in

Date: 26 June 2014.

Key words and phrases. Central simple algebras, algebraic groups, projective homogeneous varieties, Severi-Brauer varieties, Weil transfer, Chow groups and motives, canonical dimension and incompressibility. Mathematical Subject Classification (2010): 20G15; 14C25.

The author acknowledges a partial support of the French Agence Nationale de la Recherche (ANR) under reference ANR-12-BL01-0005; his work has been also supported by the start-up grant of the University of Alberta and a Discovery Grant from the National Science and Engineering Board of Canada. 
the case of a single $\mathcal{R} \mathrm{SB}$-variety. The core result producing the solution was the following statement on $p$-incompressibility of $p \mathcal{R S B}$-varieties (we refer to [11] for an introduction and further references on $p$-incompressibility, canonical $p$-dimension, Weil transfer, etc.):

Theorem 1.1 ([11, Theorem 11.2]). Any pRSB-variety is p-incompressible.

In the present paper, we generalize Theorem 1.1 in two directions. First, we replace Galois field extensions in the definition of $p \mathcal{R} \mathrm{SB}$-varieties, by separable ones. Although this generalization is not motivated anymore by study of representations, it is a natural step to do. The statement remains the same (see Theorem 2.3), as does the main outline of the proof. The main change is in the definition of a balanced algebra over a separable extension. All this is done in $\S 2$.

As a second (and principal) generalization, we establish a criterion of $p$-incompressibility of a product of $p \mathcal{R S B}$-varieties, see Corollary 3.6. The basic result here is a $p$-incompressibility criterion for a product of a $p \mathcal{R} \mathrm{SB}$-variety by an arbitrary projective homogeneous (under an action of a semisimple affine algebraic group) variety given in Theorem 3.1.

As a particular case of Corollary 3.6, we recover in $\S 4$ a (with a new proof and a simplified statement) an old result [10, Theorem 2.1] on $p$-incompressibility of products of SeveriBrauer varieties. But now we can also determine canonical $p$-dimension of any $p$-primary Weil transfer of a product of this type, see $\S 4 \mathrm{~b}$. In particular, we show that a $p$-primary Weil transfer of a $p$-incompressible product of this type is also $p$-incompressible. Moreover, the latter statement also holds for generalized Severi-Brauer varieties in place of the usual ones, see $\S 4 \mathrm{c}$.

In the last section ( $(5)$, we drop the balance assumption to do a complete analysis of a quadratic field extension. Note however, that the balanced case, motivated by the representations, also looks interesting (not only for a quadratic $L / F$ ) from the following viewpoint: for a $p \mathcal{R} \mathrm{SB}$-variety $Y$, the integer $\operatorname{cd}_{p}(Y)=\operatorname{dim} Y$ turns out to be much bigger than $\operatorname{cd}_{p}\left(Y_{L}\right)=(\operatorname{dim} Y) /[L: F]$ so that the role of the field extension and the Weil transfer shows up as crucial. In the imbalanced case however, the variety $Y_{L}$ may, for instance, happen to be $p$-incompressible (c.f. Example 5.3), trivially implying $p$-incompressibility of $Y$ itself.

Most of our terminology and notation being introduced "on the move", we only mention here that smooth projective varieties $X$ and $Y$ over $F$ are called equivalent if there exist rational maps $X \rightarrow Y$ and $Y \rightarrow X$. Equivalent varieties have the same canonical $(p$-)dimension, [11, Lemma 3.3(a)].

ACKnowledgements. I am grateful to Alexander Merkurjev and Zinovy Reichstein for useful comments on early versions of the preprint. Its current version has been prepared during my stay at the Universität Duisburg-Essen (research group of Marc Levine), under ideal work conditions.

\section{Non-Galois extensions}

In this section we generalize Theorem 1.1 to the case of an arbitrary finite separable (not necessarily Galois) field extension $L / F$ of degree a power of $p$.

Here is the "Galois to separable" generalization of [11, Lemma 11.1] (with practically the same proof). Here and everywhere else in the paper, the motive we are talking about 
are Chow motives with coefficients in $\mathbb{F}_{p}$ (the finite field of $p$ elements), see e.g. [2, §64]. Weil transfer of motives has been introduced in [5]. Corestriction of motives is from [7, $\S 3]$.

Lemma 2.1. Let $L / F$ be an arbitrary finite separable field extension and let $E / F$ be a finite Galois field extension containing $L$. For and any $m \geq 1$, let $M_{1}, \ldots, M_{m}$ be $m$ motives over $L$. Then the motive $\mathcal{R}_{L / F}\left(M_{1} \oplus \cdots \oplus M_{m}\right)$ decomposes into a direct sum

$$
\mathcal{R}_{L / F}\left(M_{1} \oplus \cdots \oplus M_{m}\right) \simeq \mathcal{R}_{L / F} M_{1} \oplus \cdots \oplus \mathcal{R}_{L / F} M_{m} \oplus N
$$

where $N$ is a direct sum of corestrictions to $F$ of motives over fields $K$ with $F \subsetneq K \subset$ E.

Let $L / F$ be a finite separable field extension. We generalize the notion of a balanced central simple $L$-algebra $D$ (which we already have in the Galois case, see $\S 1$ ). A central simple $L$-algebra $D$ is balanced, if for a Galois field extension $E / F$ containing $L$ and for any $F$-imbedding of $L$ into $E$ with an image $L_{0}$, the $L L_{0}$-algebra obtained from $D$ by the base change $L \rightarrow L_{0} \subset L L_{0}$ is Brauer-equivalent to a tensor power of $D_{L L_{0}}$. This definition does not depend on the choice of $E ; E$ can be taken to be a Galois closure of $L / F$.

From now on, we extend the notion of a $p \mathcal{R S B}$-variety, introduced in $\S 1$, by allowing separable (not necessarily Galois) Weil transfer:

Definition 2.2. Let $p$ be a prime number. An $F$-variety $Y$ is a $p \mathcal{R S B}$-variety, if there exist a finite $p$-primary separable field extension $L / F$ and a balanced division $L$-algebra $D$ of degree $p^{n}$ such that $X \simeq \mathcal{R}_{L / F} \mathrm{SB}\left(p^{i}, D\right)$ for some $i=0, \ldots, n$.

Theorem 2.3. For any prime number $p$, any $p \mathcal{R S B}$-variety is $p$-incompressible.

Proof. Let $p$ be a prime number, $L / F$ a finite separable field extension of degree a power of $p, D$ a balanced central division $L$-algebra of degree $p^{n}$ for some $n \geq 0$, and $X$ the generalized Severi-Brauer variety $\mathrm{SB}\left(p^{i}, D\right)$ of $D$ with some $i=0,1, \ldots, n$. Let us prove that the variety $\mathcal{R}_{L / F} X$, given by the Weil transfer of $X$, is $p$-incompressible.

Let $E$ be a finite Galois field extension of $F$ containing $L$ (for instance, $E$ can be a Galois closure of $L / F)$. The question on canonical $p$-dimension of $\mathcal{R}_{L / F} X$ easily reduces to the case where $[E: F]$ is also a power of $p$. Indeed, let $G$ be the Galois group of $E / F$ and let $H$ be its subgroup corresponding to $L$. Let $G^{\prime}$ be a Sylow $p$-subgroup of $G$ containing a Sylow $p$-subgroup $H^{\prime}$ of $H$. We get the following diagram of subgroups and the corresponding diagram of subfields, where the sign $p^{?}$ marks $p$-primary while the sign $p \nmid$ marks $p$-coprime indexes/degrees:

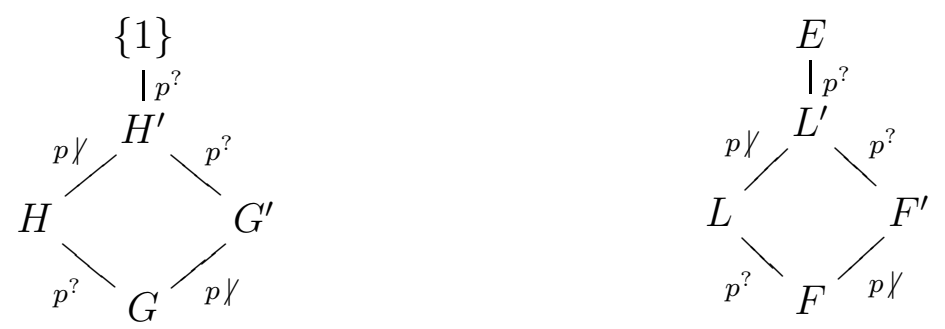

Since the degree $\left[F^{\prime}: F\right]$ is not divisible by $p$, canonical $p$-dimension of $\mathcal{R}_{L / F} X$ does not change under the base change $F^{\prime} / F$. Moreover, $\left(\mathcal{R}_{L / F} X\right)_{F^{\prime}} \simeq \mathcal{R}_{L^{\prime} / F^{\prime}}\left(X_{L^{\prime}}\right)$ because 
$L^{\prime} \simeq L \otimes_{F} F^{\prime}$. Finally, observe that the index of a $p$-primary central simple $L$-algebra is not changed under the base change $L^{\prime} / L$. In particular, $D_{L^{\prime}}$ is still a division algebra. Moreover, this central division $L^{\prime}$-algebra is still balanced (now with respect to the subfield $\left.F^{\prime} \subset L^{\prime}\right)$.

For the rest of the proof we will assume that the degree $[E: F]$ is a $p$-power. We follow the proof of Theorem 1.1, given in [11, Theorem 11.2], and only indicate the changes.

The first place where a change is needed is the place where we compute the index of $D$ over the field $L^{\prime}=F^{\prime} \otimes_{F} L$, where $F^{\prime}$ is the function field of the variety $\mathcal{R}_{L / F} \mathrm{SB}\left(p^{n-1}, D\right)$. In the case of Galois $L / F$, the Weil transfer disappears because $\mathcal{R}_{L / F} \mathrm{SB}\left(p^{n-1}, D\right)_{L}$ is the product of the generalized Severi-Brauer varieties given by the conjugate algebras. This happens because of the isomoprhism

$$
L \otimes_{F} L \simeq L \times \cdots \times L
$$

of $L$-algebras, where $L$ acts on $L \otimes L$ on the left. Note that this isomorphism is also $L$-linear for the right action of $L$ on the tensor product if one lets $L$ act on the factors of $L \times \cdots \times L$ by means of the $F$-automorphisms of $L$.

Under the assumptions of Theorem 2.3 however, $L \otimes_{F} L$ is identified with $L_{1} \times \cdots \times L_{r}$, where each $L_{i}$ is $L L_{0}$ for an appropriate (depending on $i$ ) choice of $L_{0}$ as in the definition of balanced algebra. Since $\left[L_{i}: L\right]>1$ for at least one value of $i$, we cannot avoid the Weil transfer here and may want to use the index reduction formulas for the Weil transfer of generalized Severi-Brauer varieties from [15] (see also [14]). But this is not really necessary. Indeed, since $D$ is balanced, for any $i$, the $L_{i}$-algebra in question is Brauer-equivalent to a tensor power of $D_{L_{i}}$, its index will divide $p^{n-1}$ if we extend $L$ to the function field of $\mathrm{SB}\left(p^{n-1}, D\right)$. The Weil transfer becomes rational over this extension of $L$ so that the index of $D$ won't be affected by passing to the function field of the Weil transfer.

We have explained why the index of $D_{L^{\prime}}$ is indeed $p^{n-1}$. The final adjustment we need to make, in order to adopt the proof of Theorem 1.1 to the setting of Theorem 2.3, is in the choice of the degree $p$ Galois field subextension $\tilde{L} / F$ of $L / F$. Recall that the Galois group $G$ of $E / F$ is a $p$-group. Let $H$ be its subgroup corresponding to the field $L$. By [3, Theorem 4.2.1], $H$ is contained in a normal subgroup $\tilde{H}$ of $G$ such that $[G: \tilde{H}]=p$. We take for $\tilde{L}$ the field corresponding to $\tilde{H}$ and we have to compute canonical $p$-dimension of the variety $\mathcal{R}_{L / F}(X)_{\tilde{L}}$.

The variety $\mathcal{R}_{L / F}(X)_{\tilde{L}}$ is isomorphic to the product $\prod_{\tilde{g} \in \tilde{G}} \tilde{g}\left(\mathcal{R}_{L / \tilde{L}} X\right)$, where $\tilde{G}=G / \tilde{H}$ is the Galois group of $\tilde{L} / F$. Since $D$ is balanced, this product is equivalent to its factor $\mathcal{R}_{L / \tilde{L}} X$ given by $\tilde{g}=1$. In particular, the canonical $p$-dimension of $\mathcal{R}_{L / F}(X)_{\tilde{L}}$ is given by the dimension of $\mathcal{R}_{L / \tilde{L}} X$. After this is established, the remainder of the proof of Theorem 1.1 goes through unchanged.

\section{INCOMPRESSIBILITY OF PRODUCTS}

Here is our basic result on $p$-incompressibility of products:

Theorem 3.1. Let $Y$ be a pRSB-variety (for a given prime number $p$ ) and let $Z$ be a projective homogeneous $F$-variety. The product $Y \times Z$ is $p$-incompressible provided that the varieties $Y_{F(Z)}$ and $Z_{F(Y)}$ are so. 
Proof. Let $Y:=\mathcal{R}_{L / F} \mathrm{SB}\left(p^{i}, D\right)$, where $L / F$ is a finite separable field extension of degree $p^{r}$ with $r \geq 0, D$ is a balanced central division $L$-algebra of degree $p^{n}$ with $n \geq 0$, and $i=0, \ldots, n$. Let $Z$ be a projective homogeneous $F$-variety such that the varieties $Y_{F(Z)}$ and $Z_{F(Y)}$ are $p$-incompressible. We are going to prove that the product $Y \times Z$ is $p$-incompressible.

By Theorem 2.3, the assumption requiring that $Y_{F(Z)}$ is $p$-incompressible is equivalent to the assumption that $D_{L(Z)}$ is a division algebra.

Since canonical $p$-dimension is not changed under base field extensions of degree prime to $p$ (see [12, Proposition 1.5(2)]), we may assume that there exists a finite $p$-primary Galois field extension $E / F$ containing $L$ and such that $Z_{E}$ is of inner type. This assumption allows us to apply results of [7] and $[8, \S 6]$.

For any $j=0, \ldots, n$, we set $X^{j}:=\mathrm{SB}\left(p^{j}, D\right)$ and $Y^{j}:=\mathcal{R}_{L / F} X^{j}$. We also set $X:=X^{i}$ so that $Y=\mathcal{R}_{L / F} X$.

We induct on $n$. For $n=0$ the statement is trivial. From now on, we assume that $n>0$.

For $i=n$ the statement is trivial. From now on, we assume that $i<n$.

Let $F^{\prime}$ be the function field of the variety $Y^{n-1}$. Let $L^{\prime}:=F^{\prime} \otimes_{F} L$. Since $D$ is balanced, the index of the central simple $L^{\prime}$-algebra $D_{L^{\prime}}:=D \otimes_{L} L^{\prime}=D \otimes_{F} F^{\prime}$ is $p^{n-1}$ so that there exists a central division $L^{\prime}$-algebra $D^{\prime}$ such that the algebra of $(p \times p)$-matrices over $D^{\prime}$ is isomorphic to $D_{L^{\prime}}$. We set $X^{\prime j}:=\mathrm{SB}\left(p^{j}, D^{\prime}\right), Y^{\prime j}:=\mathcal{R}_{L^{\prime} / F^{\prime}} X^{\prime j}, X^{\prime}:=X^{\prime i}$, and $Y^{\prime}:=Y^{\prime i}$. By [4] (see also [1, Theorem 7.5]) and [9, Theorem 3.8], the motive of the variety $X_{L^{\prime}}$ decomposes into a direct sum

$$
\begin{aligned}
& M\left(X_{L^{\prime}}\right) \simeq M\left(X^{\prime}\right) \oplus M\left(X^{\prime}\right)\left(p^{i+n-1}\right) \oplus \\
& \quad M\left(X^{\prime}\right)\left(2 p^{i+n-1}\right) \oplus \cdots \oplus M\left(X^{\prime}\right)\left((p-1) p^{i+n-1}\right) \oplus N,
\end{aligned}
$$

where $N$ is a direct sum of some shifts of the upper motives $U\left(X^{\prime j}\right)$ of some varieties $X^{\prime j}$ with $j<i$. Therefore, by Lemma 2.1 and [5, Theorem 5.4] (which we use just to determine the shifts), the motive of the variety $Y_{F^{\prime}}=\mathcal{R}_{L^{\prime} / F^{\prime}}\left(X_{L^{\prime}}\right)$ decomposes into a direct sum

$$
\begin{aligned}
& M\left(Y_{F^{\prime}}\right) \simeq M\left(Y^{\prime}\right) \oplus M\left(Y^{\prime}\right)\left(p^{r+i+n-1}\right) \oplus \\
& \quad M\left(Y^{\prime}\right)\left(2 p^{r+i+n-1}\right) \oplus \cdots \oplus M\left(Y^{\prime}\right)\left((p-1) p^{r+i+n-1}\right) \oplus N \oplus N^{\prime},
\end{aligned}
$$

where now $N$ is a direct sum of shifts of $U\left(Y^{\prime j}\right)$ with $j<i$, and $N^{\prime}$ is a direct sum of corestrictions of motives over fields $K$ with $F \subsetneq K \subset E$. It follows that

$$
\begin{aligned}
& M(Y \times Z)_{F^{\prime}} \simeq M\left(Y^{\prime} \times Z_{F^{\prime}}\right) \oplus M\left(Y^{\prime} \times Z_{F^{\prime}}\right)\left(p^{r+i+n-1}\right) \oplus \\
& M\left(Y^{\prime} \times Z_{F^{\prime}}\right)\left(2 p^{r+i+n-1}\right) \oplus \cdots \oplus M\left(Y^{\prime} \times Z_{F^{\prime}}\right)\left((p-1) p^{r+i+n-1}\right) \oplus N \oplus N^{\prime},
\end{aligned}
$$

with $N^{\prime}$ of the same shape as before and with $N$ being a direct sum of shifts of

$$
U\left(Y^{\prime j}\right) \otimes M(Z), \quad j<i .
$$

We claim that the variety $Y^{\prime} \times Z_{F^{\prime}}$ is $p$-incompressible by the induction hypothesis. To check the claim, we check that the varieties $Z_{F^{\prime}\left(Y^{\prime}\right)}$ and $Y_{F^{\prime}(Z)}^{\prime}$ are $p$-incompressible. To check that $Z_{F^{\prime}\left(Y^{\prime}\right)}$ is $p$-incompressible, we check that $Z$ over a lager field $F^{\prime}\left(Y^{\prime} \times Y_{F^{\prime}}\right)$ is 
so. The field $F^{\prime}\left(Y^{\prime} \times Y_{F^{\prime}}\right)=F^{\prime}(Y)\left(Y^{\prime}\right)$ is purely transcendental over $F(Y)$ and $Z_{F(Y)}$ is $p$-incompressible (this is the place in the proof of Theorem 3.1, where the assumption on $Z_{F(Y)}$ is used). Therefore $Z$ over $F^{\prime}\left(Y^{\prime} \times Y_{F^{\prime}}\right)$ is $p$-incompressible. (To see that canonical $p$-dimension of projective homogeneous varieties does not change under a purely transcendental base field extension, one may use the characterization [8, Corollary 6.2] of the canonical $p$-dimension in terms of algebraic cycles together with the fact that a purely transcendental base field extension does not affect the Chow groups.)

To check that $Y_{F^{\prime}(Z)}^{\prime}$ is $p$-incompressible, we check that $D_{L^{\prime}(Z)}^{\prime}$ is a division algebra. Since $L^{\prime}=L\left(Y^{n-1}\right)$, we have $L^{\prime}(Z)=L\left(Y^{n-1} \times Z\right)=L(Z)\left(Y^{n-1}\right)$. Since $D_{L(Z)}$ is a balanced (over $F(Z)$ ) division algebra, $D_{L(Z)\left(Y^{n-1}\right)}$ has index $p^{n-1}$. Therefore $D_{L^{\prime}(Z)}^{\prime}$ is a division algebra.

The claim being proved, it follows that

$$
\begin{aligned}
M(Y \times Z)_{F^{\prime}} & \simeq U\left(Y^{\prime} \times Z_{F^{\prime}}\right) \oplus U\left(Y^{\prime} \times Z_{F^{\prime}}\right)\left(p^{r+i+n-1}\right) \oplus \\
& U\left(Y^{\prime} \times Z_{F^{\prime}}\right)\left(2 p^{r+i+n-1}\right) \oplus \cdots \oplus U\left(Y^{\prime} \times Z_{F^{\prime}}\right)\left((p-1) p^{r+i+n-1}\right) \oplus N
\end{aligned}
$$

with $N$ having the property that no summand of its complete decomposition is isomorphic to a shift of $U\left(Y^{\prime} \times Z_{F^{\prime}}\right)$.

We want to show that the variety $Y \times Z$ is $p$-incompressible. Let $l$ be the number of those summands in the complete decomposition of the motive $U(Y \times Z)_{F^{\prime}}$, which are isomorphic to a shift of $U\left(Y^{\prime} \times Z_{F^{\prime}}\right)$. We have $1 \leq l \leq p$ and it suffices to show that $l=p$. Indeed, $l=p$ implies that the complete decomposition of $U(Y \times Z)_{F^{\prime}}$ contains the summand $U\left(Y^{\prime} \times Z_{F^{\prime}}\right)$ of $(3.2)$ with the maximal shift $(p-1) p^{r+i+n-1}$. Therefore

$$
\begin{aligned}
\operatorname{cd}_{p}(Y \times Z)=\operatorname{dim} U(Y \times Z)= & \operatorname{dim} U(Y \times Z)_{F^{\prime}} \geq \\
& \operatorname{dim} U\left(Y^{\prime} \times Z_{F^{\prime}}\right)+(p-1) p^{r+i+n-1}=\operatorname{dim}(Y \times Z) .
\end{aligned}
$$

(We refer to [6, Theorem 5.1] for the relation between canonical $p$-dimension and dimension of the upper motive of a projective homogeneous variety, used here.)

Our next claim is: $l$ divides $p$ (therefore $l=1$ or $l=p$, and we will only need to show that $l \neq 1$ ). To prove the claim, we consider the complete motivic decomposition of $Y \times Z$. It contains several shifts of $U(Y \times Z)$ (it contains one non-shifted $U(Y \times Z)$ and - as we hope - no other shifts of this motive, but we do not know whether the hope comes true by now). Let $N$ be any of the remaining (indecomposable) summands. We affirm that no summand of the complete decomposition of $N_{F^{\prime}}$ is isomorphic to a shift of $U\left(Y^{\prime} \times Z_{F^{\prime}}\right)$. Clearly, this affirmation implies the claim that $l$ divides $p$.

To prove the affirmation, let us note that $N$ can be of two alternative types. The first type is given by corestriction to $F$ of a motive over a field $K$ with $F \subsetneq K \subset E$. For such $N$, any indecomposable summand of $N_{F^{\prime}}$ is a corestriction to $F^{\prime}$ of a motive over $K^{\prime}:=K \otimes_{F} F^{\prime}$ (see [7, Proposition 3.1]) which is never isomorphic to a shift of the upper motive of a projective homogeneous $F^{\prime}$-variety.

The second type of $N$ is a shift of $U(T)$, where $T$ is a projective homogeneous variety with $\operatorname{deg} \mathrm{Ch}_{0}\left(T_{F(Y \times Z)}\right)=0$ (and with $\operatorname{deg} \mathrm{Ch}_{0}(Y \times Z)_{F(T)}=\mathbb{F}_{p}$ ). Over $F^{\prime}$ we still have $\operatorname{deg} \mathrm{Ch}_{0}\left(T_{F^{\prime}(Y \times Z)}\right)=0$ (and $\left.\operatorname{deg} \mathrm{Ch}_{0}(Y \times Z)_{F^{\prime}(T)}=\mathbb{F}_{p}\right)$ because the field extension

$$
F^{\prime}(Y \times Z) / F(Y \times Z)
$$


is purely transcendental. Any indecomposable summand of $N_{F^{\prime}}$, which is not corestriction from some $F \subsetneq K \subset E$, is a shift of $U(S)$, where $S$ is a projective homogeneous $F^{\prime}$-variety with $\operatorname{deg} \mathrm{Ch}_{0}\left(T_{F^{\prime}(S)}\right)=\mathbb{F}_{p}$. It follows that $\operatorname{deg} \mathrm{Ch}_{0}\left(S_{F^{\prime}(Y \times Z)}\right)=0$ (otherwise we would be in contradiction with $\operatorname{deg} \mathrm{Ch}_{0}\left(T_{F^{\prime}(Y \times Z)}\right)=0$ ) implying that no shift of $U(S)$ (and consequently no shift of $U(S))$ is isomorphic to $U\left(Y^{\prime} \times Z_{F^{\prime}}\right)$, see [9, Corollary 2.15] for the criterion of isomorphism for upper motives.

We proved the affirmation and the claim. This means that we only need to show that $l \neq 1$ to finish the proof of Theorem 3.1. So, we assume that $l=1$ and we look for a contradiction. By [8, Proposition 2.4], the complete decomposition of $U(Y \times Z)_{F^{\prime}}$ contains as a summand the motive $U\left(Y^{\prime} \times Z_{F^{\prime}}\right)$ shifted by the difference

$$
\operatorname{dim} U(Y \times Z)-\operatorname{dim} U\left(Y^{\prime} \times Z_{F^{\prime}}\right) .
$$

Therefore, $l=1$ implies that the above difference is 0 , and we come to

$$
\operatorname{cd}_{p}(Y \times Z)=\operatorname{dim} U(Y \times Z)=\operatorname{dim} U\left(Y^{\prime} \times Z_{F^{\prime}}\right)=d,
$$

where $d:=\operatorname{dim}\left(Y^{\prime} \times Z_{F^{\prime}}\right)=\operatorname{dim} Y^{\prime}+\operatorname{dim} Z$.

By [8, Proposition 6.1], there exist $\alpha \in \mathrm{Ch}^{d}(Y \times Z)_{F(Y \times Z)}$ and $\beta \in \mathrm{Ch}_{d}(Y \times Z)$ with $\operatorname{deg}(\alpha \cdot \beta) \neq 0 \in \mathbb{F}_{p}$. In the last formula, we consider both cycles over a common field extension of their fields of definition, before we multiply them. We use this convention below (in similar formulas on degree of products) as well.

Since $\operatorname{cd}_{p}\left(Y_{F^{\prime}}\right)=\operatorname{dim} Y^{\prime}=: d^{\prime}$, we can find $\alpha^{\prime} \in \mathrm{Ch}^{d^{\prime}}\left(Y_{F^{\prime}(Y)}\right)$ and $\beta^{\prime} \in \mathrm{Ch}_{d^{\prime}}\left(Y_{F^{\prime}}\right)$ with $\operatorname{deg}\left(\alpha^{\prime} \cdot \beta^{\prime}\right) \neq 0$. Using these $\alpha^{\prime}$ and $\beta^{\prime}$ and a rational point pt $\in Z_{F(Z)}$, we get the cycles

$$
\alpha^{\prime} \times[\mathbf{p t}] \in \mathrm{Ch}^{d}(Y \times Z)_{F^{\prime}(Y \times Z)} \quad \text { and } \quad \beta^{\prime} \times[Z] \in \mathrm{Ch}_{d}(Y \times Z)_{F^{\prime}},
$$

having the same property as $\alpha$ and $\beta$ :

$$
\operatorname{deg}\left(\left(\alpha^{\prime} \times[\mathbf{p t}]\right) \cdot\left(\beta^{\prime} \times[Z]\right)\right) \neq 0 .
$$

It follows by [8, Lemma 6.5] that one can "mix up" the old cycles with the new ones and get the relation

$$
\operatorname{deg}\left(\left(\alpha^{\prime} \times[\mathbf{p t}]\right) \cdot \beta\right) \neq 0 .
$$

Since $\alpha^{\prime} \times[\mathbf{p t}]=\left(\alpha^{\prime} \times[Z]\right) \cdot([Y] \times[\mathbf{p t}])$, the last degree relation can be rewritten as $\operatorname{deg}\left(\alpha^{\prime} \cdot \beta^{\prime \prime}\right) \neq 0$, where $\beta^{\prime \prime} \in \mathrm{Ch}_{d^{\prime}}\left(Y_{F(Z)}\right)$ is the push-forward of the product $([Y] \times[\mathbf{p t}]) \cdot \beta$ along the projection $(Y \times Z)_{F(Z)} \rightarrow Y_{F(Z)}$. Since the field extension $F^{\prime}(Y) / F(Y)$ is purely transcendental, there exists $\alpha^{\prime \prime} \in \mathrm{Ch}^{d^{\prime}}\left(Y_{F(Y)}\right)$ mapped to $\alpha^{\prime}$ under the change of field homomorphism. Changing notation, we write $\alpha^{\prime \prime}$ for the image of $\alpha^{\prime \prime}$ in $\mathrm{Ch}^{d^{\prime}}\left(Y_{F(Z)(Y)}\right)$.

The cycles $\alpha^{\prime \prime} \in \mathrm{Ch}^{d^{\prime}}\left(Y_{F(Z)(Y)}\right)$ and $\beta^{\prime \prime} \in \mathrm{Ch}_{d^{\prime}}\left(Y_{F(Z)}\right)$ thus constructed have the property $\operatorname{deg}\left(\alpha^{\prime \prime} \cdot \beta^{\prime \prime}\right) \neq 0$. It follows by $\left[8\right.$, Corollary 6.2] that $\operatorname{cd}_{p}\left(Y_{F(Z)}\right) \leq d^{\prime}$. Since

$$
d^{\prime}=\operatorname{dim} Y^{\prime}=p^{i}\left(p^{n-1}-p^{i}\right)<p^{i}\left(p^{n}-p^{i}\right)=\operatorname{dim} Y,
$$

the relation $\operatorname{cd}_{p}\left(Y_{F(Z)}\right) \leq d^{\prime}$ obtained contradicts the assumption on $p$-incompressibility of the variety $Y_{F(Z)}$.

The necessary condition for $p$-incompressibility of a product of projective homogeneous varieties, showing up in the following lemma, turns out to be sufficient in the case of the special varieties we are interested in (see Corollary 3.5): 
Lemma 3.4. For any projective homogeneous F-varieties $X$ and $Y$,

$$
\operatorname{cd}_{p}(X \times Y) \leq \operatorname{cd}_{p}(X)+\operatorname{cd}_{p}\left(Y_{F(X)}\right) .
$$

In particular, a necessary condition for p-incompressibility of $X \times Y$ is p-incompressibility of $X_{F(Y)}$ and $Y_{F(X)}$.

Proof. Again, we are using the characterization of canonical $p$-dimension of projective homogeneous varieties given in [8, Corollary 6.2]. Since canonical $p$-dimension is not changed under base field extensions of degree prime to $p$ (see [12, Proposition 1.5(2)]), we may assume that the condition of [8, Corollary 6.2] on the projective homogeneous variety is satisfied for $X$ and $Y$ : both of them become of inner type over a finite $p$-primary extension of $F$. We set $x:=\operatorname{cd}_{p}(X)$ and $y:=\operatorname{cd}_{p}\left(Y_{F(X)}\right)$. We find $\alpha_{X} \in \operatorname{Ch}^{x}\left(X_{F(X)}\right)$ and $\beta_{X} \in \mathrm{Ch}_{x}(X)$ with $\operatorname{deg}\left(\alpha_{X} \cdot \beta_{X}\right) \neq 0$. Similarly, we find $\alpha_{Y} \in \mathrm{Ch}^{y}\left(Y_{F(X)(Y)}\right)$ and $\beta_{Y} \in \mathrm{Ch}_{y}\left(Y_{F(X)}\right)$ with $\operatorname{deg}\left(\alpha_{Y} \cdot \beta_{Y}\right) \neq 0$. Let $\beta_{Y}^{\prime} \in \mathrm{Ch}_{\operatorname{dim} X+y}(X \times Y)$ be an element mapped to $\beta_{Y}$ under the surjection

$$
\mathrm{Ch}_{\operatorname{dim} X+y}(X \times Y) \rightarrow \mathrm{Ch}_{y}\left(Y_{F(X)}\right)
$$

given by the pull-back along the morphism $Y_{F(X)} \rightarrow X \times Y$ induced by the generic point of $X$. We set

$$
\alpha:=\alpha_{X} \times \alpha_{Y} \in \mathrm{Ch}^{x+y}(X \times Y)_{F(X \times Y)} \quad \text { and } \quad \beta:=\left(\beta_{X} \times[Y]\right) \cdot \beta_{Y}^{\prime} \in \mathrm{Ch}_{x+y}(X \times Y) .
$$

We have the relation $\operatorname{deg}(\alpha \cdot \beta) \neq 0$ showing that $\operatorname{cd}_{p}(X \times Y) \leq x+y$.

Corollary 3.5. For two products $X$ and $Y$ of $p \mathcal{R S B}$-varieties over $F$, their product $X \times Y$ is p-incompressible if and only if the varieties $X_{F(Y)}$ and $Y_{F(X)}$ are p-incompressible.

Proof. The "only if" part being served by Lemma 3.4, we only prove the "if" part. We write $X$ as product $X_{1} \times \cdots \times X_{r}$ of $p \mathcal{R} \mathrm{SB}$-varieties and induct on $r$. The case of $r=1$ follows from Theorem 3.1. For $r>1$, set $X^{\prime}:=X_{2} \times \cdots \times X_{r}$. To show that $X \times Y=$ $X_{1} \times\left(X^{\prime} \times Y\right)$ is $p$-incompressible, by Theorem 3.1, it suffices to check that $\left(X_{1}\right)_{F\left(X^{\prime} \times Y\right)}$ and $\left(X^{\prime} \times Y\right)_{F\left(X_{1}\right)}$ are $p$-incompressible. The assumption on $p$-incompressibility of $X_{F(Y)}$ implies (by Lemma 3.4) $p$-incompressibility of $\left(X_{1}\right)_{F\left(X^{\prime} \times Y\right)}$. And the variety $\left(X^{\prime} \times Y\right)_{F\left(X_{1}\right)}$ is $p$-incompressible by induction hypothesis.

Here is the most convenient statement to check $p$-incompressibility of a general product of $p \mathcal{R} \mathrm{SB}$-varieties. Basically, it reduces the problem to application of index reduction formulas.

Corollary 3.6. Product $X_{1} \times \cdots \times X_{r}$ of $p \mathcal{R S B}$-varieties over $F$ is $p$-incompressible if and only if $\left(X_{i}\right)_{F\left(X_{1} \times \cdots \times X_{i-1} \times X_{i+1} \times \cdots \times X_{r}\right)}$ for every $i=1, \ldots, r$, is $p$-incompressible.

\section{Particular products}

4a. Products of Severi-Brauer varieties. Applying Corollary 3.6 to a product of Severi-Brauer varieties (Weil transfer do not show up here), we get a version of $[10$, Theorem 2.1]. Note that the proof is different from the original one: it does not involve $K$-theory. 
Corollary 4.1. Let $D_{1}, \ldots, D_{r}$ be p-primary central division F-algebras. The product of their Severi-Brauer varieties is p-incompressible if and only if each $D_{i}$ remains division algebra over the function field of the product of the Severi-Brauer varieties of the remaining algebras.

Remark 4.2. By the index reduction formula for Severi-Brauer varieties, the condition on $D_{i}$ means that the index of every product of $D_{i}$ by tensor powers of the remaining algebras is $\geq$ ind $D_{i}$. With this, one may see that $D_{1}, \ldots, D_{r}$ satisfy the condition of Corollary 4.1 if and only if their nonzero Brauer classes (put in some/any order increasing the degrees) form a minimal basis in the sense of [10, Remark 2.9].

Remark 4.3. In general, the property of being $p$-incompressible for a projective homogeneous variety $X$ is weaker than the property of having indecomposable motive. However, for a generically split $X$ (i.e., for $X$ such that the motive of $X_{F(X)}$ is split, i.e., is a direct sum of shifts of the motive of a point), the above two properties are equivalent. Indeed, $M(X)$ for a generically split $X$ is a direct sum of shifts of $U(X)$ so that $M(X)=U(X)$ is and only if $\operatorname{dim} U(X)=\operatorname{dim} X$.

A product of Severi-Brauer varieties is a generically split projective homogeneous variety. Therefore the motive of a $p$-incompressible product of Severi-Brauer varieties is indecomposable.

There are numerous simplification in the proof of Theorem 3.1 when we adopt it to products of Severi-Brauer varieties. It might be therefore helpful for the reader to look at the simplified proof below before going through the actual proof of Theorem 3.1.

Proposition 4.4. Let $X$ be the product of Severi-Brauer varieties $X_{1}, \ldots, X_{r}$ over $F$ such that for every $i=1, \ldots, r$, the variety $\left(X_{i}\right)_{F\left(X_{1} \times \cdots \times X_{i-1} \times X_{i+1} \times \cdots \times X_{r}\right)}$ is $p$-incompressible. Then the motive of $X$ is p-incompressible.

Proof. Using induction on $r$, we are reduced to prove the following statement (which looks more like the statement of Theorem 3.1): the product $Y \times Z$ of a Severi-Brauer variety $Y$ by a product of Severi-Brauer varieties $Z$ is p-incompressible provided that the varieties $Y_{F(Z)}$ and $Z_{F(Y)}$ are so.

Actually, instead of being a product of Severi-Brauer varieties, $Z$ can be any generically split projective homogeneous variety: we will only use this property of $Z$ in the proof.

Now we go along the lines of the proof of Theorem 3.1, removing everything superfluous.

The variety $Y$ is the Severi-Brauer variety of a $p$-primary central division algebra $D$, say, $\operatorname{deg} D=p^{n}$. Note that $D$ remains division over $F(Z)$.

We induct on $n$. For $n=0$ the statement we are proving is trivial. We assume that $n \geq 1$ below.

Let $F^{\prime}$ be the function field of the variety $\mathrm{SB}\left(p^{n-1}, D\right)$. The index of the central simple $F^{\prime}$-algebra $D_{F^{\prime}}$ is $p^{n-1}$ so that there exists a central division $F^{\prime}$-algebra $D^{\prime}$ such that the algebra of $(p \times p)$-matrices over $D^{\prime}$ is isomorphic to $D_{F^{\prime}}$. We set $Y^{\prime}:=\operatorname{SB}\left(D^{\prime}\right)$. The motive of the variety $Y_{F^{\prime}}$ decomposes into the direct sum of $p$ summands

$$
M\left(Y_{F^{\prime}}\right) \simeq M\left(Y^{\prime}\right) \oplus M\left(Y^{\prime}\right)\left(p^{n-1}\right) \oplus M\left(Y^{\prime}\right)\left(2 p^{n-1}\right) \oplus \cdots \oplus M\left(Y^{\prime}\right)\left((p-1) p^{n-1}\right) .
$$


It follows that

$$
\begin{aligned}
M(Y \times Z)_{F^{\prime}} \simeq M\left(Y^{\prime} \times Z_{F^{\prime}}\right) \oplus M\left(Y^{\prime} \times Z_{F^{\prime}}\right)\left(p^{n-1}\right) \oplus \\
M\left(Y^{\prime} \times Z_{F^{\prime}}\right)\left(2 p^{n-1}\right) \oplus \cdots \oplus M\left(Y^{\prime} \times Z_{F^{\prime}}\right)\left((p-1) p^{n-1}\right) .
\end{aligned}
$$

We claim: the decomposition (4.5) is complete, i.e., the motive of the variety $Y^{\prime} \times Z_{F^{\prime}}$ is indecomposable (see Remark 4.3), and that - by the induction hypothesis. To check the claim, we check that the varieties $Z_{F^{\prime}\left(Y^{\prime}\right)}$ and $Y_{F^{\prime}(Z)}^{\prime}$ are $p$-incompressible. This is done precisely as in the proof of Theorem 3.1.

Now we know that (4.5) is the complete decomposition and we want to show that the motive of $Y \times Z$ is indecomposable. In other words, we want to show that

$$
U(Y \times Z)=M(Y \times Z),
$$

or - equivalently - that $U(Y \times Z)_{F^{\prime}}$ contains all the $p$ indecomposable summands of the decomposition (4.5).

Let $l$ be the number of summand in the complete decomposition of the motive $U(Y \times$ $Z)_{F^{\prime}}$ (all of them are automatically isomorphic to a shift of $M\left(Z_{F^{\prime}}\right)$ ). We have $1 \leq l \leq p$ and all we want to show is $l=p$.

It is now very easy to see that $l$ divides $p$. Indeed, since the variety $Y \times Z$ is generically split, every summand of its complete motivic decomposition is a shift of $U(Y \times Z)$. Therefore $l$ divides $p$, as claimed, and we only need to show that $l \neq 1$.

So, we assume that $l=1$ and we look for a contradiction. Clearly, $l=1$ implies that

$$
\operatorname{cd}_{p}(Y \times Z)=\operatorname{dim} U(Y \times Z)=\operatorname{dim} M\left(Y^{\prime} \times Z_{F^{\prime}}\right)=d,
$$

where $d:=\operatorname{dim} Y^{\prime}+\operatorname{dim} Z=p^{n-1}-1+\operatorname{dim} Z$. By [8, Proposition 6.1], there exist $\alpha \in \operatorname{Ch}^{d}(Y \times Z)_{F(Y \times Z)}$ and $\beta \in \mathrm{Ch}_{d}(Y \times Z)$ with $\operatorname{deg}(\alpha \cdot \beta) \neq 0 \in \mathbb{F}_{p}$. Starting from this point, the proof of Proposition 4.4 ends precisely as the proof of Theorem 3.1. Note that in (3.3) we will have $i=0$ here.

\section{4b. Weil transfer of products of Severi-Brauer varieties.}

Corollary 4.6. Let $L / F$ be a p-primary separable field extension and let $X$ be a product over $L$ of Severi-Brauer varieties of some balanced p-primary division L-algebras. Then $\operatorname{cd}_{p}\left(\mathcal{R}_{L / F} X\right)=[L: F] \cdot \operatorname{cd}_{p}(X)$. In particular, $\mathcal{R}_{L / F} X$ is $p$-incompressible provided that $X$ is so.

Proof. Taking a minimal basis of the subgroup in $\operatorname{Br}(L)$ generated by the algebras (note that any $L$-algebra representing an element of this subgroup is balanced), consider the product $X^{\prime}$ of their Severi-Brauer varieties. The variety $X^{\prime}$ is $p$-incompressible and equivalent to $X$. Moreover, its Weil transfer $\mathcal{R}_{L / F} X^{\prime}$ is equivalent to $\mathcal{R}_{L / F} X$. Therefore we reduced the proof of the first statement of Corollary 4.6 to the proof of the second one. The second statement follows directly from Corollary 3.6.

4c. Weil transfer of products of generalized Severi-Brauer varieties. The second conclusion of Corollary 4.6 also holds for generalized Severi-Brauer varieties. Again, the result is an immediate consequence of Corollary 3.6: 
Corollary 4.7. Let $L / F$ be a p-primary separable field extension and let $X$ be a product over $L$ of some generalized Severi-Brauer varieties of some balanced p-primary division $L$-algebras. Then $\mathcal{R}_{L / F} X$ is p-incompressible if (and only if) $X$ is so.

\section{QuAdRATIC EXTENSIONS}

Let $L / F$ be a separable quadratic field extension and let $D$ be a central division $L$ algebra of a 2-primary index. In this section, we determine canonical 2-dimension of the variety $\mathcal{R}_{L / F} \mathrm{SB}(D)$ without imposing any restrictions on the conjugate algebra $g(D)$, where $g$ is the non-trivial element of $G:=\operatorname{Gal}(L / F)$. We will provide two different recipes; which of them has to be applied depends on a property of the group $A$ generated by the Brauer classes of $D$ and $g(D)$. Since the case of cyclic $A$ has been already treated, we assume that $A$ is not cyclic. Therefore $A / 2 A \simeq \mathbb{Z} / 2 \mathbb{Z} \oplus \mathbb{Z} / 2 \mathbb{Z}$ with $g$ acting by exchanging the summands.

Let $\alpha \in A \backslash 2 A$ be an element of the smallest index. Let $D_{\alpha}$ be a central division $L$-algebra representing $\alpha$. If the image of $\alpha$ in $A / 2 A$ is not invariant under the action of $G$, then the images of $\alpha$ and $g(\alpha)$ form a minimal basis of $A / 2 A$ in the sense of [10, Remark 2.9]. It follows that the variety $\left(\mathcal{R}_{L / F} \mathrm{SB}\left(D_{\alpha}\right)\right)_{L} \simeq \mathrm{SB}\left(D_{\alpha}\right) \times \mathrm{SB}\left(g\left(D_{\alpha}\right)\right)$ is 2incompressible. Therefore the variety $\mathcal{R}_{L / F} \mathrm{SB}\left(D_{\alpha}\right)$ is 2-incompressible as well. Since $\alpha$ and $g(\alpha)$ generate $A$, the varieties $\mathcal{R}_{L / F} \mathrm{SB}(D)$ and $\mathcal{R}_{L / F} \mathrm{SB}\left(D_{\alpha}\right)$ are equivalent. So, $\mathrm{cd}_{2} \mathcal{R}_{L / F} \mathrm{SB}(D)=\mathrm{cd}_{2} \mathcal{R}_{L / F} \mathrm{SB}\left(D_{\alpha}\right)$ and we get that

$$
\operatorname{cd}_{2} \mathcal{R}_{L / F} \mathrm{SB}(D)=\operatorname{dim} \mathcal{R}_{L / F} \mathrm{SB}\left(D_{\alpha}\right)=2(\text { ind } \alpha-1)
$$

Now we assume that the image of $\alpha$ in $A / 2 A$ is invariant under $G$. This means that this image is equal to the element $(1,1) \in \mathbb{Z} / 2 \mathbb{Z} \oplus \mathbb{Z} / 2 \mathbb{Z}$. Let $\beta \in A$ be an element of the smallest index with the property that its image in $A / 2 A$ is outside of the subgroup generated by the image of $\alpha$. We claim that in this case

$$
\operatorname{cd}_{2} \mathcal{R}_{L / F} \mathrm{SB}(D)=\operatorname{dim} \mathcal{R}_{L / F} \mathrm{SB}\left(D_{\beta}\right)=2(\operatorname{ind} \beta-1)
$$

Since the image of $\beta$ in $A / 2 A$ is $(1,0)$ or $(0,1)$, it is not invariant under $G$. Moreover, $A$ is generated by $\beta$ and $g(\beta)$. It follows that the variety $\mathcal{R}_{L / F} \mathrm{SB}(D)$ is equivalent to $\mathcal{R}_{L / F} \mathrm{SB}\left(D_{\beta}\right)$, where $D_{\beta}$ is a central division $L$-algebra representing $\beta$. To prove the claim, it suffices to prove that the variety $\mathcal{R}_{L / F} \mathrm{SB}\left(D_{\beta}\right)$ is 2 -incompressible. It is so because the $F\left(\mathcal{R}_{L / F} \mathrm{SB}\left(D_{\alpha}\right)\right)$-variety

$$
\left.\mathcal{R}_{L / F} \mathrm{SB}\left(D_{\beta}\right)_{F\left(\mathcal{R}_{L / F} \mathrm{SB}\left(D_{\alpha}\right)\right)} \simeq \mathcal{R}_{L\left(\mathcal{R}_{L / F} \mathrm{SB}\left(D_{\alpha}\right)\right) / F\left(\mathcal{R}_{L / F} \mathrm{SB}\left(D_{\alpha}\right)\right)} \mathrm{SB}\left(\left(D_{\beta}\right)_{L\left(\mathcal{R}_{L / F}\right.} \mathrm{SB}\left(D_{\alpha}\right)\right)\right)
$$

is 2-incompressible. Indeed, $g\left(D_{\beta}\right)_{L\left(\mathcal{R}_{L / F} \mathrm{SB}\left(D_{\alpha}\right)\right)}$ is Brauer-equivalent to a tensor power of $\left(D_{\beta}\right)_{L\left(\mathcal{R}_{L / F} \mathrm{SB}\left(D_{\alpha}\right)\right)}$ so that Theorem 1.1 applies. On the other hand, $\left(D_{\beta}\right)_{L\left(\mathcal{R}_{L / F} \mathrm{SB}\left(D_{\alpha}\right)\right)}$ is still a division algebra by the Schofield-van den Bergh index reduction formula [16] (see also [13]), because

$$
\operatorname{ind}\left(D_{\beta} \otimes D_{\alpha}^{\otimes i}\right) \geq \text { ind } D_{\beta}
$$

for any $i$ by the minimality of ind $\beta=$ ind $D_{\beta}$.

We finish this section by examples where the above recipes apply.

Example 5.3. Let $l$ be a field of characteristic $\neq 2$, let $L$ be the rational function field over $l$ in variables $x, y, x^{\prime}, y^{\prime}$, let $g$ be the $l$-automorphism of $L$ exchanging $x$ with $x^{\prime}$ and $y$ 
with $y^{\prime}$, and let $F$ be the subfield of $L$ consisting of the elements fixed by $g$. The variety $\mathcal{R}_{L / F} \mathrm{SB}(D)$, where $D$ is the quaternion $L$-algebra $(x, y)$, is 2 -incompressible by formula (5.1) with $D_{\alpha}=D$. Note that in this example the algebras $D$ and $g(D)$ are, informally speaking, "completely independent".

In the next example, $D$ and $g(D)$ will be "partially dependent". To get it, we replace $F$ and $L$ by the rational function fields

$$
F\left(s_{1}, s_{2}, t_{1}, t_{2}\right) \text { and } L\left(s_{1}, s_{2}, t_{1}, t_{2}\right)
$$

in some variables $s_{1}, s_{2}, t_{1}, t_{2}$. For $D:=C \otimes(x, y)$ with $C:=\left(s_{1}, t_{1}\right) \otimes\left(s_{2}, t_{2}\right)$, the variety $\mathcal{R}_{L / F} \mathrm{SB}(D)$ is 2-incompressible by formula (5.2) with $D_{\alpha}=(x, y) \otimes\left(x^{\prime}, y^{\prime}\right)$ and $D_{\beta}=D$. Note that the non-zero elements of the group $A$ in this situation are the Brauer classes of the index 8 conjugate algebras $C \otimes(x, y)$ and $C \otimes\left(x^{\prime}, y^{\prime}\right)$ and the index 4 invariant algebra $(x, y) \otimes\left(x^{\prime}, y^{\prime}\right)$; besides, $2 A=0$.

\section{REFERENCES}

[1] Chernousov, V., Gille, S., and Merkurjev, A. Motivic decomposition of isotropic projective homogeneous varieties. Duke Math. J. 126, 1 (2005), 137-159.

[2] Elman, R., Karpenko, N., and Merkurjev, A. The algebraic and geometric theory of quadratic forms, vol. 56 of American Mathematical Society Colloquium Publications. American Mathematical Society, Providence, RI, 2008.

[3] Hall, JR., M. The theory of groups. The Macmillan Co., New York, N.Y., 1959.

[4] Karpenko, N. A. Cohomology of relative cellular spaces and of isotropic flag varieties. Algebra $i$ Analiz 12, 1 (2000), 3-69.

[5] Karpenko, N. A. Weil transfer of algebraic cycles. Indag. Math. (N.S.) 11, 1 (2000), 73-86.

[6] Karpenko, N. A. Canonical dimension. In Proceedings of the International Congress of Mathematicians. Volume II (New Delhi, 2010), Hindustan Book Agency, pp. 146-161.

[7] Karpenko, N. A. Upper motives of outer algebraic groups. In Quadratic forms, linear algebraic groups, and cohomology, vol. 18 of Dev. Math. Springer, New York, 2010, pp. 249-258.

[8] Karpenko, N. A. Sufficiently generic orthogonal Grassmannians. J. Algebra 372 (2012), 365-375.

[9] Karpenko, N. A. Upper motives of algebraic groups and incompressibility of Severi-Brauer varieties. J. Reine Angew. Math. 677 (2013), 179-198.

[10] Karpenko, N. A., And Merkurjev, A. S. Essential dimension of finite p-groups. Invent. Math. 172, 3 (2008), 491-508.

[11] Karpenko, N. A., And Reichstein, Z. A numerical invariant for linear representations of finite groups. Linear Algebraic Groups and Related Structures (preprint server) 534 (2014, May 15, revised: 2014, June 18), 24 pages.

[12] Merkurjev, A. S. Essential dimension. In Quadratic Forms - Algebra, Arithmetic, and Geometry, vol. 493 of Contemp. Math. Amer. Math. Soc., Providence, RI, 2009, pp. 299-326.

[13] Merkurjev, A. S., Panin, I. A., And Wadsworth, A. R. Index reduction formulas for twisted flag varieties. I. K-Theory 10, 6 (1996), 517-596.

[14] Merkurjev, A. S., Panin, I. A., And Wadsworth, A. R. Index reduction formulas for twisted flag varieties. II. $K$-Theory 14, 2 (1998), 101-196.

[15] Saltman, D. J. The Schur index and Moody's theorem. K-Theory 7, 4 (1993), 309-332.

[16] Schofield, A., And VAn Den Bergh, M. The index of a Brauer class on a Brauer-Severi variety. Trans. Amer. Math. Soc. 333, 2 (1992), 729-739.

Mathematical \& Statistical Sciences, University of Alberta, Edmonton, CANADA

E-mail address: karpenko at ualberta.ca, web page: www.ualberta.ca/ ${ }^{k a r p e n k o}$ 